\title{
Are Non-dysplastic Crypts with Corrupted Shapes the Initial Recordable Histological Event in the Development of Sporadic Conventional Adenomas?
}

\author{
CARLOS A. RUBIO ${ }^{1}$ and PETER T. SCHMIDT ${ }^{2}$ \\ ${ }^{1}$ Gastrointestinal and Liver Research Laboratory, Department of Pathology, and ${ }^{2}$ Department of Medicine (Solna), \\ Center for Digestive Diseases, Karolinska Institute and University Hospital, Stockholm, Sweden
}

\begin{abstract}
Background: Colonic crypts with normal epithelial lining displaying corrupted shapes (called non-dysplastic crypts with corrupted shapes, NDCs) were earlier recorded underneath the adenomatous glands of conventional colon adenomas in rats. Aim: To assess the frequency of NDCs in clinical sporadic conventional (tubular/villous) adenomas. Materials and Methods: NDCs found underneath the adenomatous epithelium in 255 sporadic conventional adenomas removed at endoscopy were classified into four groups: i) With fission distortions, ii) with length distortions, iii) with outline distortions, and iv) with axial polarity distortions. In 22 controls, the colonic mucosa proximal or distal to surgically removed colonic adenocarcinomas was scrutinized for NDCs. Results: Nearly three-quarters of the sporadic conventional adenomas investigated here had three or more NDCs underneath the adenomatous tissue, those with $\geq 4$ NDCs being more frequent (46.3\%) than those having 1, 2 or 3 NDCs $(p<0.05)$. Nineteen out of the 22 control colon segments had normal crypts and the remaining three had occasional NDCs (mean=3.7, range $=2-5)$. Conclusion: NDCs were found underneath the adenomatous glands in all 255 sporadic conventional adenomas. Occasionally, NDCs were present in the mucosa of the stalk of pediculated conventional adenomas. The absence of adenomatous tissue in NDCs of the stalk should rule out the possibility that the adenomatous tissue on top had directly orchestrated the development of NDCs below. Moreover, NDCs rarely occurred in controls. Accordingly, NDCs emerge as a genuine phenomenon of crypt
\end{abstract}

Correspondence to: Carlos A. Rubio, MD, Ph.D., Gastrointestinal and Liver Pathology Research Laboratory, Department of Pathology, Karolinska Institute and University Hospital, 17176, Stockholm, Sweden. Tel: +46 851774527, Fax: +46851774524. E-mail: Carlos.Rubio@ki.se

Key Words: Colon, crypt deformity, non-dysplastic crypts with corrupted shapes, conventional adenomas. deformation in sporadic conventional adenomas. Considering that human colonic crypts typically divide at most once or twice during a lifetime, with an average crypt cycle length of 36 years, the accumulation of NDCs underneath sporadic conventional adenomas is remarkable. In light of these considerations, it is suggested that these putative mutated NDCs may represent the initial histological recordable event heralding the development of sporadic conventional adenomas in the human colon.

The colonic mucosa is constantly challenged by a series of carcinogenic factors able to generate mucosal foci of mutated dysplastic cells called adenomas, lesions that may eventually progress to invasive carcinoma. For many years, the general view was that the vast majority of colorectal carcinomas (CRC) in humans evolved from sporadic conventional (tubular or villous) adenomas via the adenoma-carcinoma pathway (1). More recently, serrated hyperplastic polyps, sessile serrated polyps and traditional serrated adenomas have been incorporated into the pathway of colorectal carcinogenesis (2, 3 ). It has been estimated that about $30 \%$ of all CRCs progress via the serrated pathway (4). In a study of all sporadic colorectal adenomas registered at the Karolinska University Hospital between 1993 and $2000(n=3135)$, we found that 93\% were conventional adenomas and the remaining $7 \%$ were traditional serrated adenomas (serrated and microtubular) (5). Hyperplastic polyps and sessile serrated polyps were not included in the study evaluation. From the above, it might be deduced that conventional adenoma continues to be the most commonly reported histological phenotype of sporadic colorectal adenomas.

The main risks for the growth of a sporadic conventional adenoma include family history, advanced age, male sex, lifestyle, natural exposure to dietary/environmental carcinogens, genetic differences, obesity, type 2 diabetes, the colonic microbiome (6) and the disintegration of the subepithelial macrophage barrier against the gut microbiota (7). Progression from conventional adenoma to carcinoma is 

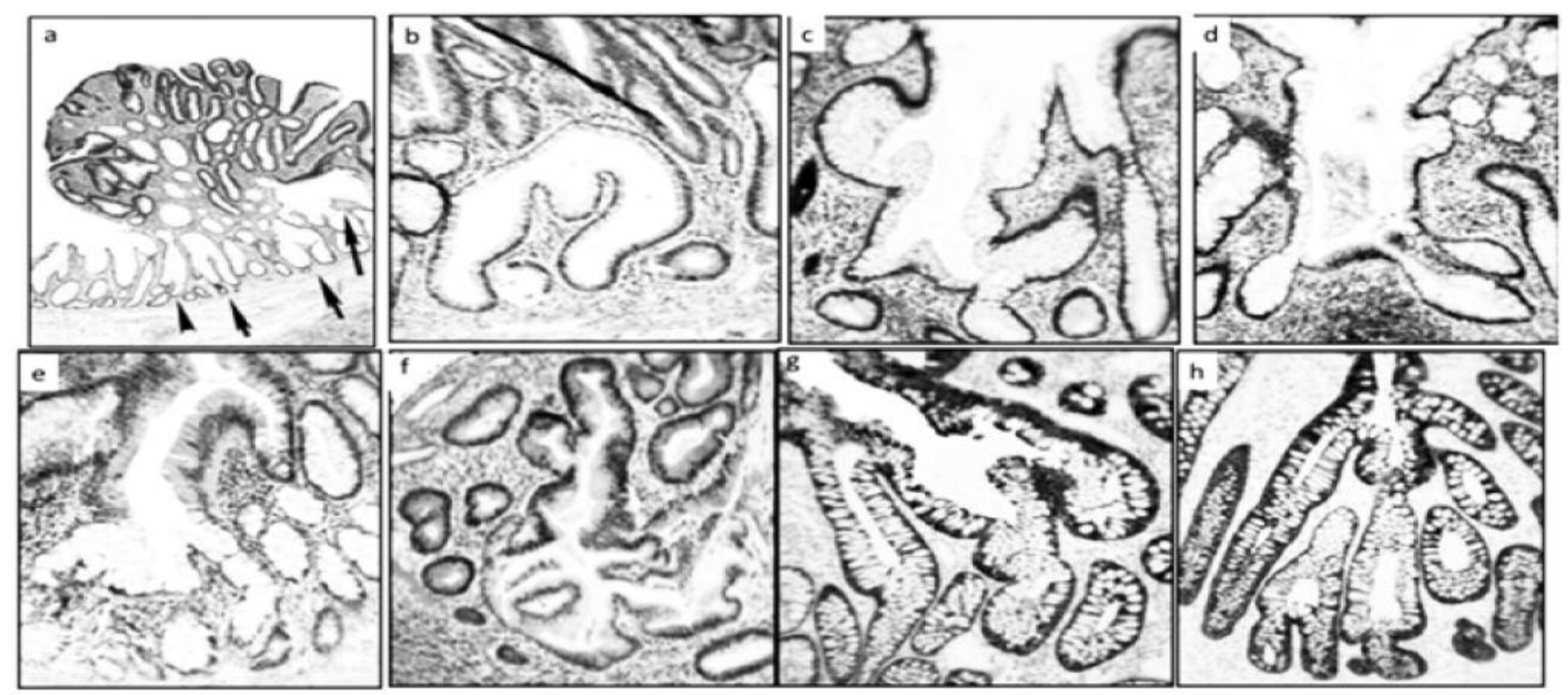

Figure 1. Group I: Fission distortions exhibited by nondysplastic corrupted colonic crypts (NDCs) underneath conventional (tubular/villous) colonic adenomas. a: NDC (arrows) underneath a tubular adenoma $(H \& E, \times 4) . b$ : NDC showing tripartite cryptal bifurcation $(H \& E, \times 10) . c$ and d: Examples of NDC with multipartite asymmetric bifurcation $(H \& E, \times 10)$. e and $f$ : Examples of NDC showing corrupted crypt bifurcations $(H \& E$, $\times 4) . g$ and $h$ : Examples of NDC underneath conventional adenoma (MNF 116 cytokeratin staining, $\times 10)$.

triggered by the accumulation of molecular aberrations and by epigenetic modifications $(8,9)$.

The normal colonic mucosa is built with a single-cell epithelial layer exhibiting finger-like invaginations or folds called crypts. When histological sections are cut perpendicular to the mucosal surface, the crypts appear as a 'row of test tubes' with parallel, tightly packed crypts 'resting' on the muscularis mucosae. According to histology books and medical publications, the aforementioned crypt architecture is consistent throughout the normal colon, with the exception of the innominate grooves, where crypts connect to a single lumen (10-12). Albeit crypts replicate by symmetric fission, starting at their base, until two identical individual crypts are generated (10-12), crypt branching is rarely found in fixed preparations. In conventional adenomas, the adenomatous glands are initially found at the luminal aspect of the lesion, progressing downwards to eventually replace the normal colorectal crypts underneath, a mechanism referred to a top-down replacement $(13,14)$.

In a previous study, we observed that colonic crypts in colonic adenomas adopted irregular shapes but retained the normal epithelial lining (15). More recently, the review of colectomy sections from Sprague-Dawley rats treated with the carcinogen dimethylhydrazine disclosed haphazardly distributed crypts with irregular shapes lined with indigenous normal epithelium. These crypts were called non-dysplastic crypts with corrupted shapes (NDCs) (16). Notably, NDCs were also found below the adenomatous tissue of dimethylhydrazine-induced conventional adenomas (16).

In the present communication, we reviewed a relatively large cohort of clinical sporadic conventional adenomas in order to evaluate the shape of the crypts with irregular shapes lined with indigenous normal epithelium below the adenomatous tissue.

\section{Materials and Methods}

The studied material comprised sections from 255 endoscopically removed sporadic conventional adenomas. Sections were retrieved from the files of the Gastrointestinal Research Laboratory of the Department of Pathology, Karolinska Institute and University Hospital. The sections were stained with hematoxylin and eosin (H\&E). The H\&E sections from the 255 sporadic conventional adenomas were scrutinized for crypts exhibiting irregular shapes with normal epithelium lining, located below the adenomatous tissue. These crypts are referred to using the acronym NDCs and those with dysplastic epithelium in the adenomatous tissue as DCs.

In 55 out of the 255 cases, extra sections were immunostained with a broad-spectrum cytokeratin marker which stains high-and low-molecular weight cytokeratins 5, 6, 8, 17 and probably 19, (MNF 116; Dako Automation, Glostrup, Denmark) to highlight epithelial structures exclusively.

Controls. Histological sections from 22 colonic segments, proximal to or distal a surgically removed colonic adenocarcinoma, were retrieved from the files of the Gastrointestinal Research Laboratory and also reviewed. Using a $10 \times$ ocular and a $10 \times$ objective (aperture 


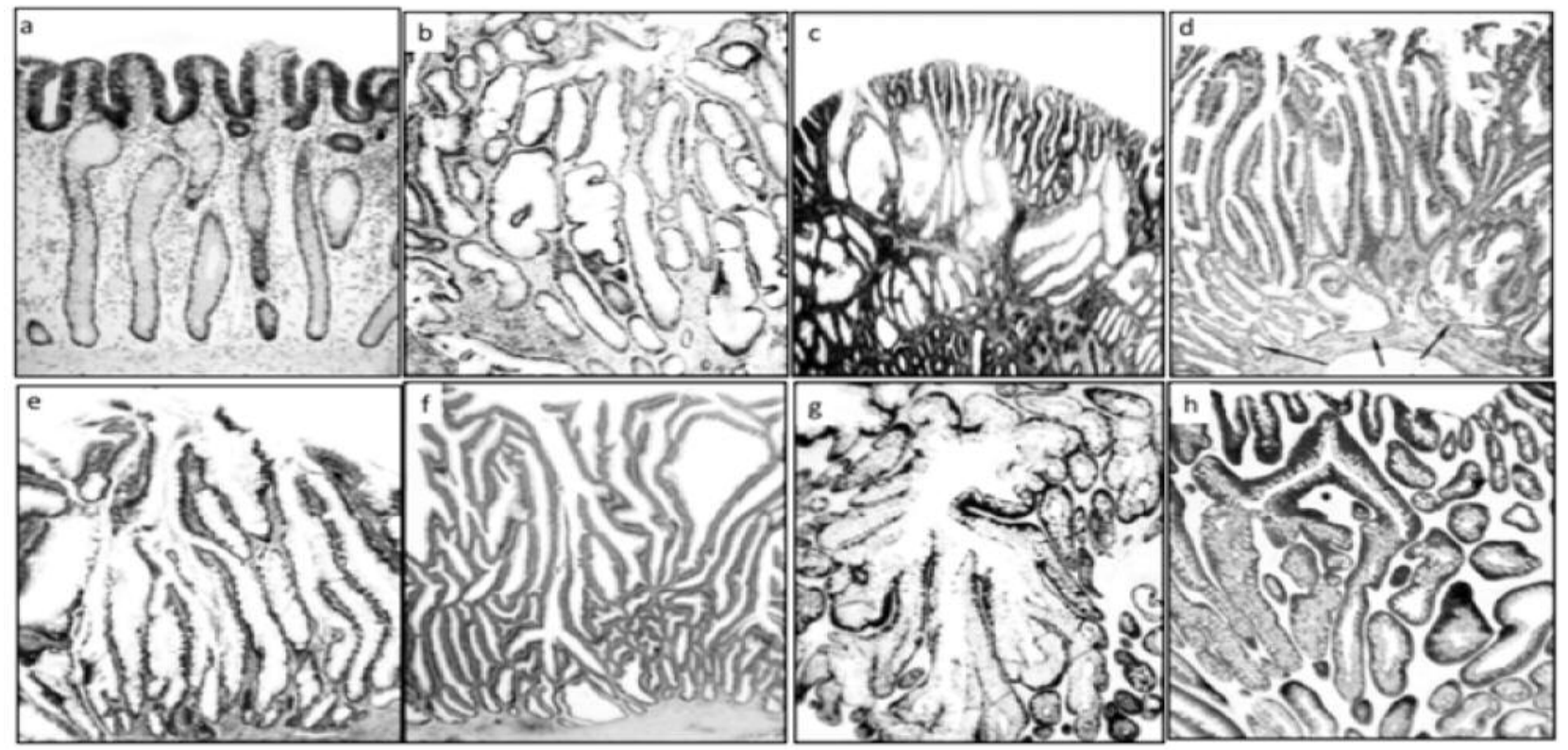

Figure 2. Group II: Length distortions exhibited by nondysplastic corrupted colonic crypts (NDCs) underneath conventional (tubular/villous) colonic adenomas. a: Hyperplastic NDC underneath a conventional adenoma $(H \& E, \times 10)$. b: Hyperplastic NDC underneath a conventional adenoma $(H \& E, \times 10)$. c: Another example of hyperplastic NDC underneath a conventional adenoma $(H \& E, \times 4) . d-f:$ Examples of NDC underneath villous adenomas $(H \& E, \times 4) . g$ and $h:$ Hyperplastic NDC with multipartite asymmetric bifurcations underneath conventional adenoma (MNF 116 cytokeratin staining, $\times 20$ and $\times 10$, respectively).

$0.45)$, the number of crypts present in 15 continuous fields of view, corresponding to $30 \mathrm{~mm}$ of colonic mucosa, was registered for each of the control segments.

In 27 out of the 255 conventional adenomas, the stalk covered with normal-looking mucosa was also analyzed for crypts with irregular shapes despite exhibiting normal epithelium lining.

NDCs were classified into the following groups: Group I: NDCs with fission distortions, i.e. exhibiting two or multiple nonidentical crypt fissions (Figure 1); group II: NDCs with length distortions, i.e. hyperplasic crypts being more than twice the length of a normal crypt (Figure 2); group III: NDCs with outline distortions, i.e. crypts with multi-lobate outlines or cystic dilatations (Figure 3); and, group IV: NDCs with axial-polarity distortions in relationship to the muscularis mucosae, i.e. 'riverdelta' pattern, serpentine crypts, intercommunicating horizontal crypts, inverted L-shaped or ring-shaped horizontal crypts in 'indian files' (Figure 4).

NDCs with fission aberrations were regarded a priori as having the lowest degree of crypt disarray, and NDCs with axial-polarity aberrations as having the highest degree of crypt pattern aberration. Adenomas having two or more NDC phenotypes coexisting underneath the adenomatous tissue were classified according to the NDCs showing the highest degree of architectural disarray.

Statistical analysis. The non-parametric Kruskal-Wallis test was applied to compare difference between groups. Statistical significance was defined as $p<0.05$.

This study was approved by The Regional Ethical Review Board in Stockholm (no. 2018/688-32).

\section{Results}

Out of the 255 conventional adenomas, 170 (66.7\%) were tubular adenomas and the remaining 85 (33.3\%) were villous adenomas. Out of the 170 tubular adenomas, $133(78.2 \%)$ showed low-grade dysplasia, and the remaining $37(21.8 \%)$ high-grade dysplasia. Of the 85 villous adenomas, 48 (56.5\%) showed low-grade dysplasia and the remaining 37 (43.5\%) high-grade dysplasia.

As shown in Table I, NDCs were classified according to the corrupted crypt showing the highest degree of architectural disarray. Non-significant differences between the four groups were recorded $(p=0.12)$.

Table II shows the number of NDCs below the basal aspect of adenomatous tissue or intercalated with DCs in the 255 conventional adenomas. Notably, nearly three-quarters of the conventional adenomas investigated here had three or more NDCs underneath the adenomatous tissue, conventional adenomas having four or more NDCs below being the most frequent $(46.3 \%)$, followed by those having three NDCs $(24.7 \%)$, two NDCs $(23.1 \%)$, and one NDCs $(5.9 \%)$. The difference in frequency between conventional adenomas with four or more NDCs, and the other three groups was significant $(p<0.05)$.

Controls. A total of 8,580 crypts present in the 15 continuous fields of view/segment (or $30 \mathrm{~mm} /$ segment) were reviewed 


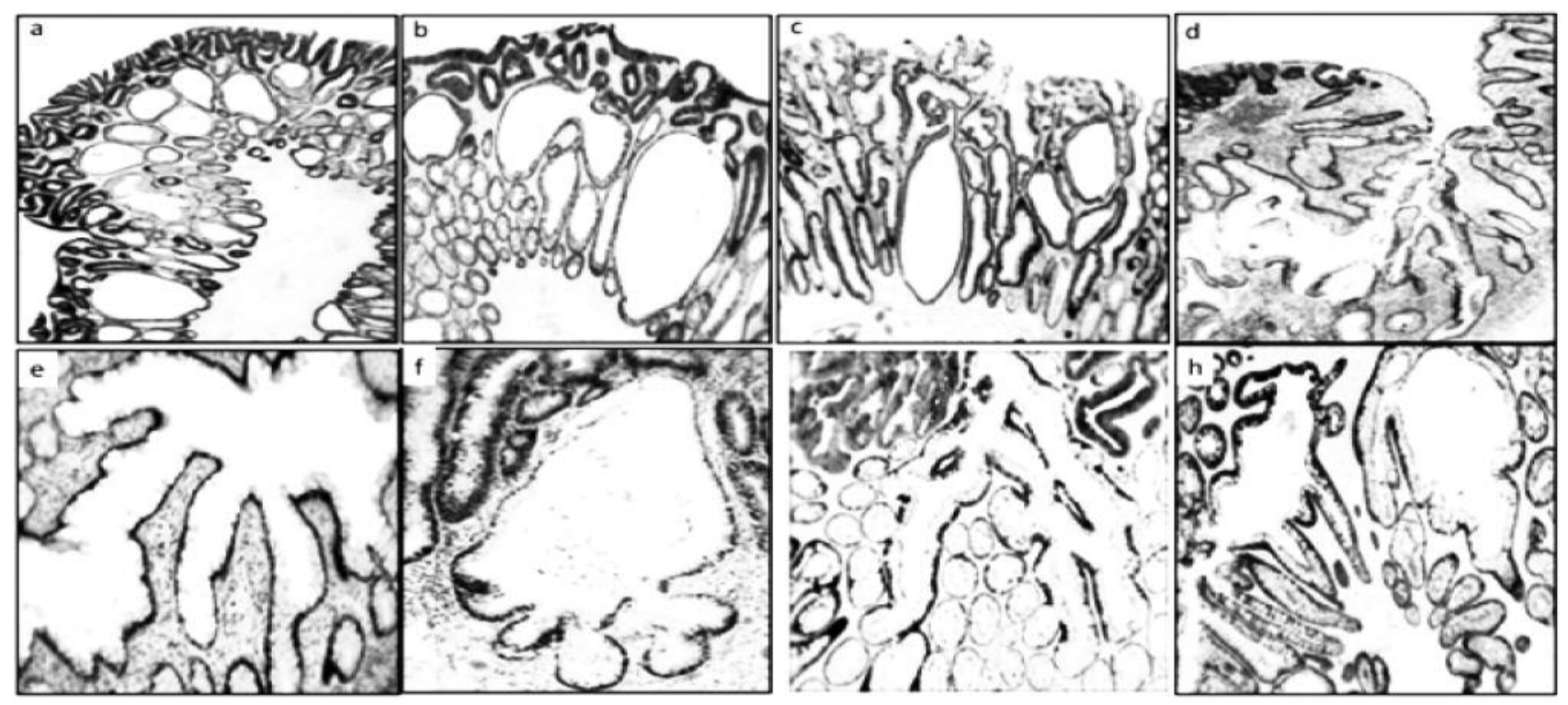

Figure 3. Group III: Outline distortions exhibited by nondysplastic corrupted colonic crypts (NDCs) underneath conventional (tubular/villous) colonic adenomas. a-c: Cystically dilated NDC underneath a conventional adenoma $(H \& E, \times 10)$. $d-f: N D C$ with multi-lobate outlines $(H \& E, \times 4$, $\times 10$ and $\times 10$, respectively). $g$ : NDC with multi-lobate outlines underneath conventional adenoma (MNF 116 cytokeratin staining, $\times 10)$. $h:$ NDC with multi-lobate outlines underneath conventional adenoma (MNF 116, ×10).
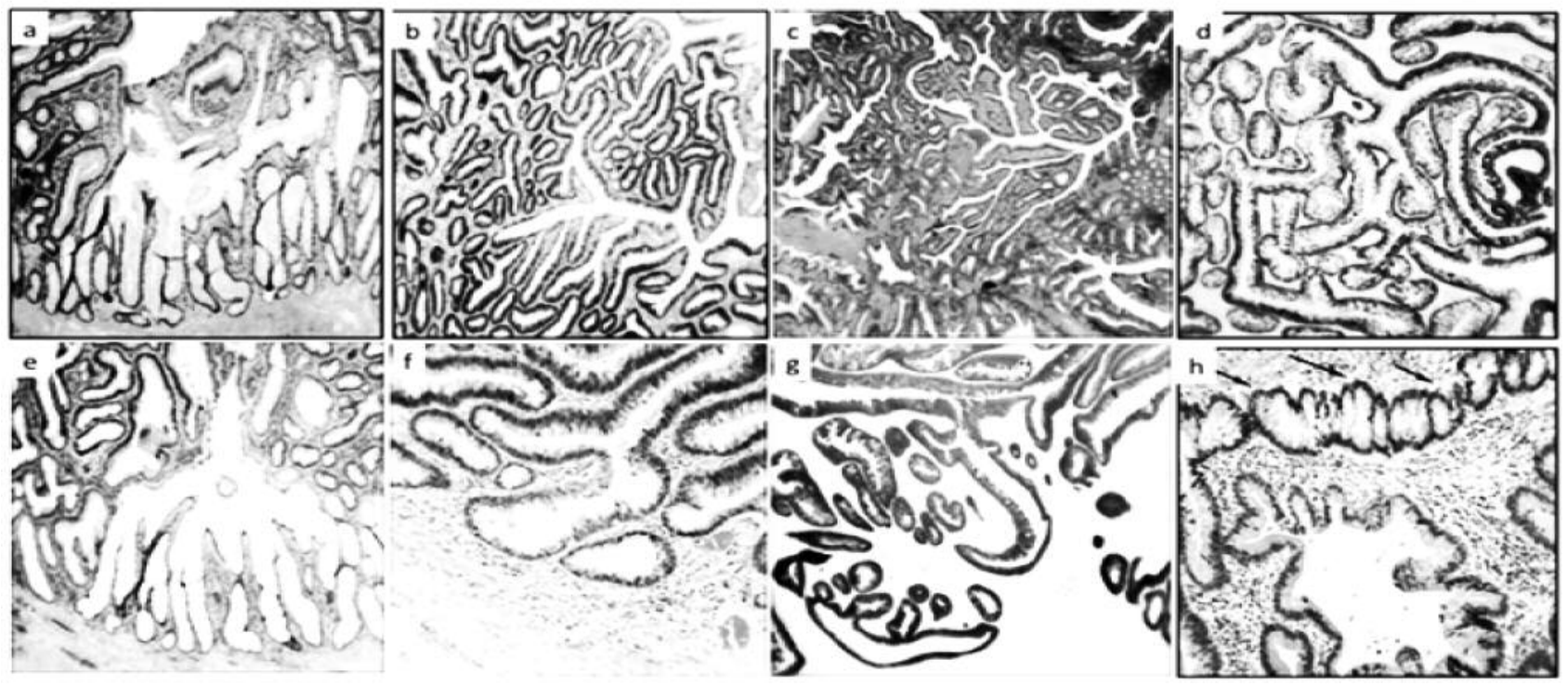

Figure 4. Group IV: Axial-polarity distortions exhibited by nondysplastic corrupted colonic crypts (NDCs) underneath conventional (tubular/villous) colonic adenomas. $a$ - $h$ : Examples of NDC with axial-polarity distortions $(a, b, e: H \& E \times 4 ; c: H \& E \times 2 ; d: M N F 116$ cytokeratin staining, $\times 4 ; f$ and $h: H \& E \times 10 ; g: M N F 116, \times 4)$. Note river-delta pattern in c-e, and horizontal crypts in 'indian files' (arrows) in $h$.

in the 22 controls (mean $=390$ crypts/segment, range $=382$ 408). Normal-shaped crypts lined with normal epithelium were found in 19 colonic segments, and occasional NDCs in the remaining three segments (mean=3.7 NDCs, range=2-5).

\section{Discussion}

In the present survey, we analyzed the morphological spectrum of NDCs found underneath sporadic conventional 
Table I. The frequency of histological groups of non-dysplastic corrupted colonic crypts (NDCs) found underneath 255 colorectal conventional adenomas. Groups I to IV are listed according to increased degree of architectural disarray.

\begin{tabular}{lccc}
\hline & & \multicolumn{2}{c}{ NDCs present, $\mathrm{n}(\%)$} \\
\cline { 3 - 4 } Group & Histological aberration & Conventional adenoma $(\mathrm{n}=255)$ & Colon mucosa (pedicle) $(\mathrm{n}=27)$ \\
\hline I & With fission aberrations & $76(29.8 \%)$ & $27(100 \%)$ \\
II & With length distortions & $62(24.3 \%)$ & $0(0 \%)$ \\
III & With outline distortions & $51(20.0 \%)$ & $0(0 \%)$ \\
IV & With axial-polarity distortions & $66(25.9 \%)$ & $0(0 \%)$ \\
Total & & $255(100 \%)$ & $27(100 \%)$ \\
\hline
\end{tabular}

adenomas. Various NDC phenotypes were frequently detected underneath the adenomatous tissue albeit occasionally interspersed with the adenomatous tissue. Nearly three-quarters of the sporadic conventional adenomas investigated here had three or more NDCs underneath the adenoma; those with four or more NDCs were most frequently encountered. In contrast, the normal mucosa from colonic segments proximal or distal to surgically removed colon cancer (control material) revealed normal-shaped crypts lined with normal epithelium in $86 \%$ of the segments, and occasional NDCs in the remaining 14\%. Thus, the NDCs found underneath adenomas emerges as a genuine phenomenon of crypt deformation in sporadic conventional adenomas. Notably, in the mucosa of the stalk of pediculated sporadic conventional adenomas, occasional NDCs were found. Accordingly, the absence of adenomatous tissue in NDCs of the stalk should rule out the possibility that the neoplastic tissue on top had directly orchestrated the development of NDCs.

The DCs found near the muscularis mucosae might mirror NDCs that were replaced by the dysplastic epithelium growing in a top-down manner (13), implying that NDCs might act as scaffold templates during top-down dysplastic cell replacement (16).

At this stage in the discussion it seems pertinent to refer to current knowledge concerning crypt fission and molecular signals that influence the shape of the crypts. Park et al. demonstrated that crypts clonally multiply through crypt fission when mutated at the glucose-6-phosphate dehydrogenase locus, and suggested that tumor initiation depends on a high fission rate of mutated crypts (17). Adenomatous polyposis coli $(A P C)$ and WNT signaling regulator control colonic crypts by adjusting their differentiation via crypt stem-cell adhesion through cadherin- $\beta$-catenin signaling (18). APC mutations were found in the top half of the neoplastic crypts, as well as in the surrounding dysplastic crypts, but not in the normalappearing bottom half of the same crypt. Thus, the NDCs underneath the adenomatous glands of sporadic conventional
Table II. The number of non-dysplastic corrupted colonic crypts (NDCs) found intercalated or below the adenomatous tissue with dysplastic corrupted colonic crypts in 255 conventional (tubular and villous) adenomas.

\begin{tabular}{lc}
\hline NDC frequency & No. of adenomas, $(\%)$ \\
\hline 1 & $15(5.9 \%)$ \\
2 & $59(23.1 \%)$ \\
3 & $63(24.7 \%)$ \\
$\geq 4$ & $118(46.3 \%)$ \\
Total & $255(100 \%)$ \\
\hline
\end{tabular}

adenomas reported here might have developed due to mutations in the $A P C$ gene via the cadherin- $\beta$-catenin axis.

The crucial question is: What are the morphogenetic mechanisms that induce crypts lined with normal epithelium underneath sporadic conventional adenomas to adopt corrupted shapes? In this context, it should be understood that morphogenesis represents the ability of a system to change its form. Jagan et al. demonstrated that the formation of colorectal crypts is regulated by phosphatase and tensin homolog deleted on chromosome 10 , a protein encoded by the PTEN gene (19). In addition, in three-dimensional studies, Georgescu et al. found that NHERF1 protein, a $\mathrm{Na}^{+} / \mathrm{H}^{+}$ exchanger regulatory factor, controls human colonic gland morphogenesis (20). Thus, the disparate phenotypes of NDCs underneath the adenomatous glands of sporadic conventional adenomas shown here might have been generated by cryptal morphogenesis signals such as NHERF1 and PTEN.

Considering that human colonic crypts typically divide at most once or twice during a lifetime, with an average crypt cycle length of 36 years (21), the accumulation of NDCs beneath conventional adenomas found here is remarkable. In light of these considerations, it is suggested that these putative mutated NDCs may be the initial histological recordable event heralding the development of conventional adenomas not only in rats (22), but also in the human colon. 


\section{References}

1 Jackman RJ and Mayo CW: The adenoma-carcinoma sequence in cancer of the colon. Surg Gynecol Obstet 93: 327-330, 1951.

2 Longacre TA and Fenoglio-Preiser CM: Mixed hyperplastic adenomatous polyps/serrated adenomas. A distinct form of colorectal neoplasia. Am J Surg Pathol 14: 524-537, 1990.

3 O'Brien MJ and Gibbons D: The adenoma-carcinoma sequence in colorectal neoplasia. Surg Oncol Clin N Am 5: 513-530, 1996.

4 O'Brien MJ, Zhao Q and Yang S: Colorectal serrated pathway cancers and precursors. Histopathology 66: 49-65, 2015.

5 Rubio CA: Colorectal adenomas: Time for reappraisal. Pathol Res Pract 198: 615-620, 2002.

6 Rabeneck L, Horton S and Zauber AG: Colorectal Cancer. In: Gelband H, Jha P, Sankaranarayanan R and Horton S (eds.) Cancer: Disease Control Priorities, Third Edition (Volume 3). Washington (DC): The International Bank for Reconstruction and Development. The World Bank; Chapter 6, pp. 344-366, 2015.

7 Rubio CA and Schmidt PT: Severe defects in the macrophage barrier to gut microflora in inflammatory bowel disease and colon cancer. Anticancer Res 38: 3811-3815, 2018.

8 Yamagishi H, Kuroda H, Imai Y and Hiraishi H: Molecular pathogenesis of sporadic colorectal cancers. Chin J Cancer 6: 35-44, 2016.

9 Shu XS, Zhao Y, Sun Y, Zhong L, Cheng Y, Zhang Y, Ning K, Tao Q, Wang Y and Ying Y: The epigenetic modifier PBRM1 restricts the basal activity of the innate immune system by repressing retinoic acid-inducible gene-I-like receptor signalling and is a potential prognostic biomarker for colon cancer. J Pathol 244: 36-48, 2018.

10 Dahl J and Greenson JK: Colon. In: Histology for Pathologists. Mills SE (eds). Lippincott Williams \& Wilkins. Third edition. Philadelphia, PA, USA. Chapter 25, pp. 629-630, 2007.

11 Ross MH, Reith EJ and Romrell LJ: Large Intestine. In: Histology. A Text and Atlas. Revised Edition. Ross MH, Reith EJ and Romrell LJ (eds.).Williams and Wilkins, Baltimore, MD, USA. pp. 440-441, 1989.

12 Levine DS and Haggitt RC: Normal histology of the colon. Am J Surg Pathol 13: 966-984,1989.

13 Shih IM, Wang TL, Traverso G, Romans K, Hamilton SR, BenSasson S, Kinzler KW and Vogelstein B: Top-down morphogenesis of colorectal tumors. Proc Natl Acad Sci USA 98: 2640-2645, 2001.
14 Preston SL, Wong WM, Chan AO, Poulsom R, Jeffery R, Goodlad RA, Mandir N, Elia G, Novelli M, Bodmer WF, Tomlinson IP and Wright NA: Bottom-up histogenesis of colorectal adenomas: origin in the monocryptal adenoma and initial expansion by crypt fission. Cancer Res 63: 3819-3825, 2003.

15 Rubio CA: Neoplasias of the colorectal crypts. Anticancer Res 25: 4551-4558, 2005.

16 Rubio CA: Corrupted colonic crypt fission in carcinogen-treated rats. PLoS One 12: e0172824, 2017.

17 Park HS, Goodlad RA and Wright NA: Crypt fission in the small intestine and colon. A mechanism for the emergence of G6PD locus-mutated crypts after treatment with mutagens. Am J Pathol 147: 1416-1427, 1995.

18 Sumigray KD, Terwilliger M and Lechler T: Morphogenesis and compartmentalization of the intestinal crypt. Dev Cell 45: 183197, 2018.

19 Jagan IC, Deevi RK, Fatehullah A, Topley R, Eves J, Stevenson $\mathrm{M}$, Loughrey $\mathrm{M}$, Arthur $\mathrm{K}$ and Campbell FC: PTEN phosphatase-independent maintenance of glandular morphology in a predictive colorectal cancer model system. Neoplasia 15: 1218-1230, 2013.

20 Georgescu MM, Cote G, Agarwal NK and White CL: NHERF1/EBP50 controls morphogenesis of 3D colonic glands by stabilizing PTEN and ezrin-radixin-moesin proteins at the apical membrane. Neoplasia 16: 365-374, 2014.

21 Baker AM, Cereser B, Melton S, Fletcher AG, Rodriguez-Justo M, Tadrous PJ, Humphries A, Elia G, McDonald SA, Wright NA, Simons BD, Jansen M and Graham TA: Quantification of crypt and stem cell evolution in the normal and neoplastic human colon. Cell Rep 8: 940-7, 2014.

22 Rubio CA: Are corrupted non-dysplastic colonic crypts the first histological event in experimental colonic carcinogenesis? Anticancer Res 37: 2265-2268, 2017.
Received May 23, 2018

Revised August 6, 2018

Accepted August 7, 2018 